\title{
Moment Tensor Analysis of Acoustic Emissions Induced by Laboratory-based Hydraulic Fracturing in Granite,
}

\section{$\operatorname{AUTHOR}(\mathrm{S}):$}

Yamamoto, Kazune; Naoi, Makoto; Chen, Youqing; Nishihara, Kengo; Yano, Shunsuke; Kawakata, Hironori; Akai, Takashi; Kurosawa, Isao; Ishida, Tsuyoshi

\section{CITATION:}

Yamamoto, Kazune ...[et al]. Moment Tensor Analysis of Acoustic Emissions Induced by Laboratory-based Hydraulic Fracturing in Granite,. Geophysical Journal International 2019, 216(3): 1507-1516

\section{ISSUE DATE:}

2019-03

URL:

http://hdl.handle.net/2433/263338

\section{RIGHT:}

This article has been accepted for publication in 'Geophysical Journal International' (c) The Author(s) 2019. Published by Oxford University Press on behalf of The Royal Astronomical Society. All rights reserved. 


\section{Moment tensor analysis of acoustic emissions induced by laboratory-based hydraulic fracturing in granite}

\author{
Kazune Yamamoto, ${ }^{1}$ Makoto Naoi, ${ }^{1}$ Youqing Chen, ${ }^{2}$ Kengo Nishihara, ${ }^{1}$ Shunsuke Yano, ${ }^{1}$ \\ Hironori Kawakata, ${ }^{3}$ Takashi Akai, ${ }^{4}$ Isao Kurosawa ${ }^{4}$ and Tsuyoshi Ishida ${ }^{1}$ \\ ${ }^{1}$ Department of Civil and Earth Resources Engineering, Graduate School of Engineering, Kyoto University, Kyoto Daigaku Katsura Cluster-C, Nishikyo-ku, \\ Kyoto615-8540, Japan.E-mail:naoi.makoto.4z@kyoto-u.ac.jp \\ ${ }^{2}$ Department of Energy Science and Technology, Graduate School of Energy Science, Kyoto University, Yoshida-Honmachi, Sakyo-ku, Kyoto 606-8501, Japan \\ ${ }^{3}$ College of Science and Engineering, Ritsumeikan University, 1-1-1 Noji Higashi, Kusatsu 525-8577, Japan \\ ${ }^{4}$ Japan Oil, Gas and Metals National Corporation, 1-2-2 Hamada, Mihama-ku, Chiba-city, Chiba 261-0025, Japan
}

Accepted 2018 November 20. Received 2018 October 19; in original form 2018 May 30

\begin{abstract}
SUMMARY
Moment Tensors of hydraulically induced AEs: Hydraulic fracturing is an important technique in the development of enhanced geothermal systems and unconventional resources. Although the fracture modes induced by hydraulic fracturing influence the recovery efficiency of the resources, the current understanding of this relationship is insufficient. In this study, we considered the acoustic emissions (AEs) induced during hydraulic fracturing under uniaxial loading conditions in the laboratory, and applied a moment tensor analysis by carefully correcting the coupling condition and directivity of AE transducers. Experiments were conducted for two types of Kurokami-jima granite samples: those with a rift plane perpendicular (Type $\mathrm{H}$ ) or parallel (Type V) to the expected direction of fracture propagation (i.e. along the loading axis). In the experiments, both sample types experienced a significant number of shear, tensile and compressive events. The dominant fracture mode for Type $\mathrm{H}$ samples is found to be tensile events in which the fracture plane is parallel to the loading axis, whereas for Type $\mathrm{V}$ samples, shear events are dominant. This difference suggests that the dominant fracture modes induced by hydraulic fracturing are highly dependent on the relationship between the direction of fracture propagation and orientation of pre-existing weak planes.
\end{abstract}

Key words: Fracture and flow; Earthquake source observations; Induced seismicity; Fractures, faults, and high strain deformation zones.

\section{INTRODUCTION}

Hydraulic fracturing is commonly used to increase the permeability of reservoir rocks in enhanced geothermal systems (EGSs) and unconventional reservoirs. The fracture modes are expected to be tensile because of the stress condition around the fracturing hole (e.g. Hubbert \& Willis 1957; Yew \& Weng 2014), whereas many previous studies have reported that shear events are dominant for the microseismic events induced in actual production fields (Horálek et al. 2010; Maxwell \& Cipolla 2011; Šilený et al. 2014; Staněk \& Eisner 2017) and in situ experiments (Kwiatek et al. 2018). Although some recent studies reported that significant non-double couple components were obtained for seismic events induced by hydraulic fracturing (Vavryčuk 2002; Silený et al. 2009; Baig \& Urbancic 2010; Julian et al. 2010), a seismic network in such production fields is often insufficient to constrain such components (Nolen-Hoeksema \& Ruff 2001), resulting in an insufficient understanding of the dominant fracture modes that are induced by hydraulic fracturing.
Because the modes of hydraulically induced fractures are likely to influence the recovery efficiency of the resources, it is vital to derive appropriate estimations and predictions. For example, Šilený et al. (2014) pointed out the importance of determining the occurrence of tensile events in EGS sites because such events are expected to make a strong contribution to increases in permeability in geothermal reservoirs. In both EGS and unconventional resource developments, proppants (small particles of sand or ceramics) are injected into induced fractures to prevent the closure of the fractures, thus maintaining high permeability for longer periods. Because the proppants may be able to penetrate into tensile cracks easier than shear cracks, the generation of tensile events is thought to be more desirable than shear events (Maxwell \& Cipolla 2011). The dominant modes of induced fractures depend on, for example, the stress conditions, dominant orientation of pre-existing weak planes and viscosity of the fracturing fluid (Ishida et al. 2004; Fischer \& Guest 2011; Shimizu et al. 2011). It is necessary to investigate the relation between the fracturing condition and dominant modes of induced fractures to ensure a better fracturing design. 


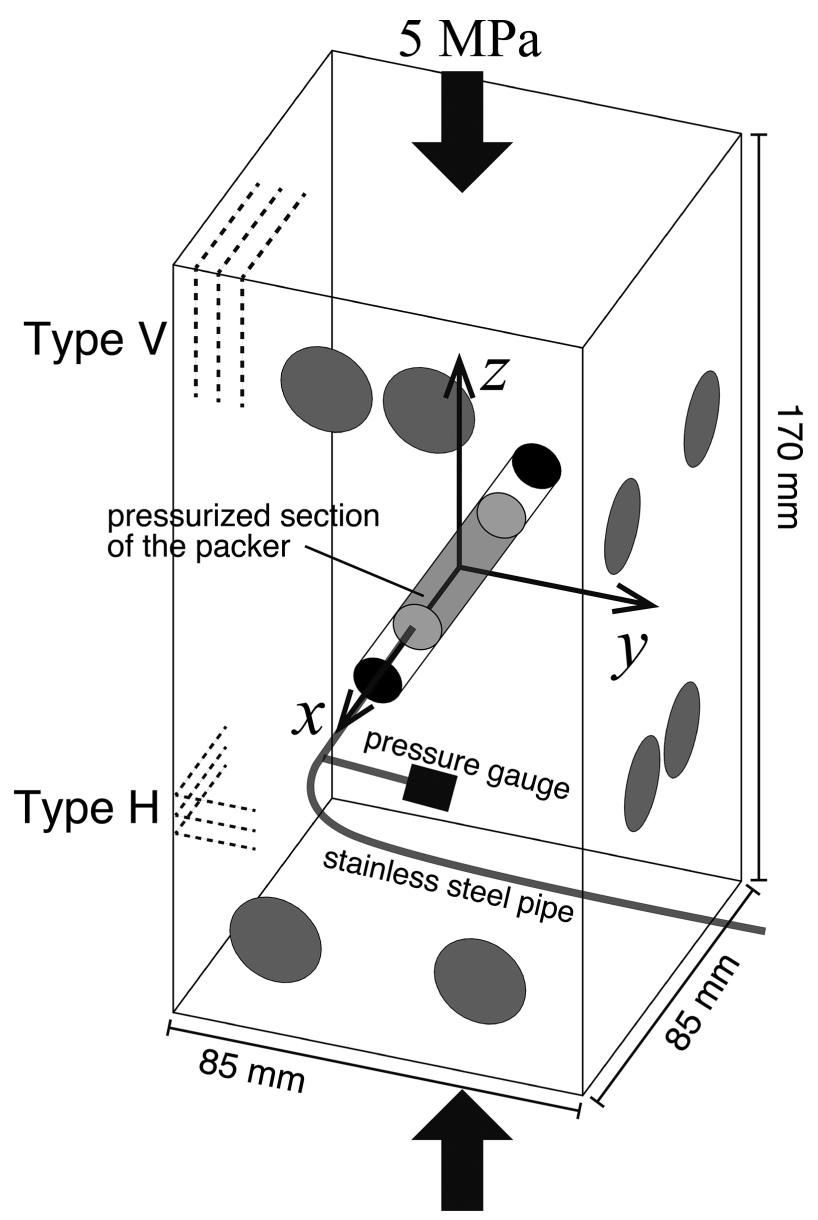

Figure 1. Sample size, position of AE transducer, loading direction and the coordinate system used in this study. The origin of the coordinate system corresponds to the centre of the specimen. The black circle represents a wellbore of $10 \mathrm{~mm}$ diameter. A packer was inserted along the $x$-axis from the positive side. Fluid pressure near the packer is measured by the pressure gauge at the entrance of the hole. Grey circles represent the positions of the $\mathrm{AE}$ transducers (shown only on the front side for clarity). Modified from Naoi et al. (2018).

In this study, we applied a moment tensor analysis to the acoustic emission (AE) data obtained in hydraulic fracturing experiments by Naoi et al. (2018) using Kurokami-jima granite samples. The experiments were conducted under uniaxial loading conditions by using samples in which the rift plane (the plane within which most of the microcracks exist; e.g. Sano et al. 1992) was either perpendicular (Type $\mathrm{H}$ specimen) or parallel (Type V specimen) to the expected direction of fracture propagation (i.e. direction of the loading axis). The obtained moment tensors showed that tensile events were dominated for Type $\mathrm{H}$, whereas shear events were dominated for Type V. This indicates that the dominant modes of fractures induced by hydraulic fracturing significantly depend on the dominant orientation of pre-existing weak planes.

\section{EXPERIMENTAL SET-UP}

In this section, we briefly explain the experimental set-up. Detailed information on the experiments was reported by Naoi et al. (2018).

The experiments were conducted using seven blocks of Kurokami-jima granite (KJG05-11) with dimensions of $85 \mathrm{~mm} \times 85 \mathrm{~mm} \times 170 \mathrm{~mm}$ (Fig. 1). Throughout this study, we use the Cartesian coordinates, where the $x$-axis is taken as the axis parallel to the wellbore, the $z$-axis is taken as parallel to the longitudinal direction of the block and the $y$-axis is taken as orthogonal to the $x$ - and $z$-axes. Based on the difference of $P$-wave velocities along the $x$-, $y$ - and $z$-axes (Section 3), Naoi et al. (2018) estimated the orientation of rift planes and classified the blocks into two types (Fig. 1): Type V (KJG05, 07, 10), in which the rift plane is parallel to the $x-z$ plane (i.e. vertical rift plane in the experimental setting) and Type $\mathrm{H}(\mathrm{KJG} 06,08,09,11)$, in which the rift plane is parallel to the $x-y$ plane (horizontal rift plane).

Fracturing was conducted under uniaxial loading of $5 \mathrm{MPa}$ along the $z$-axis. A wellbore of $10 \mathrm{~mm}$ in diameter was drilled at the centre of each block. A packer with a $30 \mathrm{~mm}$ pressurizing section sealed with O-rings at each end was inserted into the wellbore to inject a fracturing fluid. An acrylic resin (methyl methacrylate, MMA; viscosity $0.80 \mathrm{mPa}$ s at room temperature) containing a fluorescent compound was used as the fracturing fluid. After fracturing, the penetrated resins were solidified by heating, and the blocks were sliced to observe the resins delineating the induced fractures. Naoi et al. (2018) reported the details of the fluorescence observations. The MMA was provided from a cylindrical piston to the packer, which was pushed by the pressure of water injected from a syringe pump at a constant flow rate of $2 \mathrm{~cm}^{3} \mathrm{~min}^{-1}$. The wellbore pressure was measured by a pressure gauge near the packer (Fig. 1), and the data were recorded continuously at $100 \mathrm{kHz}$ sampling.

\section{MEASUREMENT OF ACOUSTIC EMISSIONS}

As shown in Fig. 1, 16 AE transducers (Physical Acoustic Corp. R15 sensor; operating frequency range $50-200 \mathrm{kHz}$ ), which can be used to receive and transmit waves, were attached to each specimen using thermofusible wax. During the experiments, signals were continuously recorded at $10 \mathrm{MHz}$ sampling using a 14-bit analogue-todigital converter (PXIe-5170R; National Instruments Corp.) after amplification by $52 \mathrm{~dB}$ and the application of a $0.02-3.00 \mathrm{MHz}$ bandpass filter. The maximum range of the analogue-to-digital converter was set to $2 \mathrm{~V}$ in peak-to-peak voltage.

From these data, we created an AE catalogue containing the hypocentre coordinates and relative magnitudes. AE events were identified using a method based on the ratios of the short-term and long-term averages (Allen 1978). For each cut-out waveform, the $P$-wave arrival time was automatically detected using the algorithm of Takanami \& Kitagawa (1988), and their hypocentres were located using a least-squares algorithm in which the $P$-wave anisotropy was considered (Rothman et al. 1974). The $P$-wave velocity $V_{\mathrm{P}}$ (and also $S$-wave velocity $V_{\mathrm{S}}$ ) was measured along the $x, y$ and $z$-axes before drilling the fracturing hole. Although the measurement was conducted under a condition without loading, the influence of $5 \mathrm{MPa}$ loading in the experimental condition is very limited because of the low pressure. Actually, we could not find significant velocity change for the data obtained in the pulse radiation test (Section 4.2), which was conducted under a $5 \mathrm{MPa}$ load to estimate AE sensor coupling. In the iterative hypocentre-determination procedure, we removed arrival times that had large residuals using a bi-weight function. From the results, we selected 'well-located events' satisfying the following conditions: (1) eight or more arrival times remained after the final iteration and (2) the maximum standard error was less than $40 \mathrm{~mm}$ among the three principal axes of an error ellipsoid calculated from the variance-covariance matrix. We then 
(a)

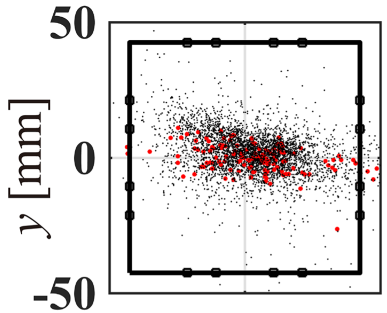

(b)

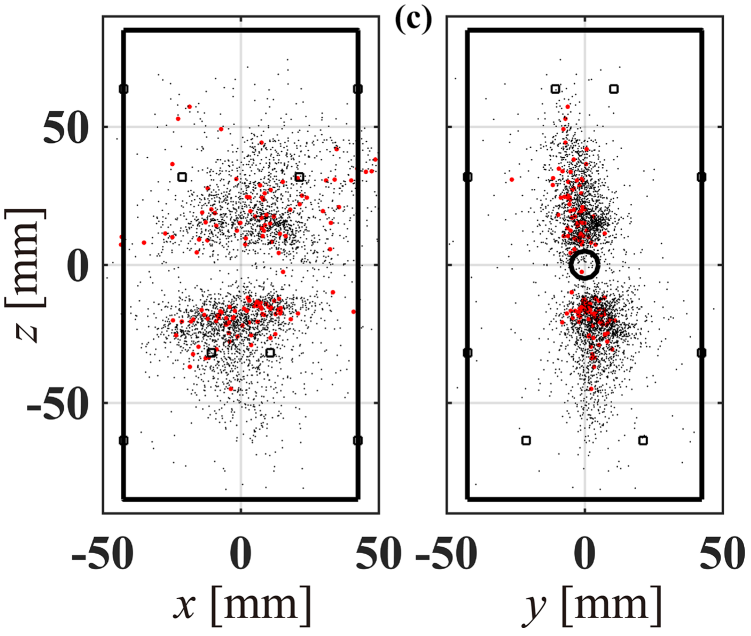

Figure 2. AE hypocentre distribution for KJG07. Relocation results obtained by the double-difference algorithm are shown: projections to (a) $x-y$ plane, (b) $x-z$ plane and (c) $y-z$ plane. The bold, black rectangle represents the size of the sample. Small rectangles show the sensor positions. Red dots represent AEs whose moment tensors were determined in Section 4 (only 142 events that have VR $\geq 50$ per cent are plotted, where VR is the variance reduction defined by eq. 6 ).

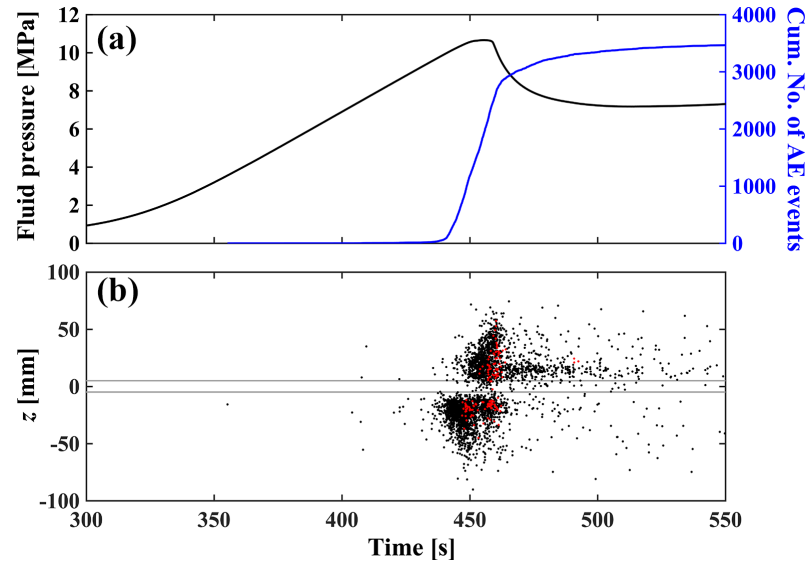

Figure 3. (a) Wellbore pressure history (black line) and cumulative number of well-located AEs as a function of time (blue line). (b) Time-position plot along the $z$-axis for the AEs relocated by the double-difference method. Red dots represent AEs whose moment tensors were determined in Section 4 (only events satisfying VR $\geq 50$ per cent are plotted).

relocated the events using the double-difference algorithm (Waldhauser \& Ellsworth 2000). Details of the catalogue development were reported by Naoi et al. (2018).

Fig. 2 represents the AE hypocentre distribution obtained for experiment KJG07 (Type V). Fig. 3 represents the AE activity and fluid pressure history of this experiment. The total duration of the experiment was $1054 \mathrm{~s}$ from the start of fluid injection to the end of
$\mathrm{AE}$ data recording, although the figure is focused on the $250 \mathrm{~s}$ interval around the breakdown point. The breakdown (a sudden drop in fluid pressure due to fracturing) occurred at $t=455 \mathrm{~s} \mathrm{(Fig.} \mathrm{3a).}$ The AE activity started before the breakdown and the hypocentres expanded outwards with time from the wellbore (Fig. 3b). The hypocentres exhibit a bi-wing distribution along the $z$-direction from the wellbore (Fig. 2c), which is consistent with the fluid penetration region observed after the fracturing experiment (Naoi et al. 2018). This distribution along the loading axis is consistent with the direction of fractures expected from the conventional theory of hydraulic fracturing (e.g. Hubbert \& Willis 1957). The features described above occurred in all seven granite samples (Naoi et al. 2018).

\section{MOMENT TENSOR INVERSION FOR AE EVENTS}

We estimated seismic moment tensors for the AEs from the polarities of the $P$-wave first motions and their amplitudes. The directivity of an $\mathrm{AE}$ transducer is generally unknown, and its sensitivity depends on the specific coupling condition between the specimen and the sensor. This makes it difficult to invert the moment tensor, which requires wave amplitude information. To overcome these problems, we measured the directivity and estimated the relative sensitivity of individual $\mathrm{AE}$ transducers using the following procedure.

\subsection{Directivity of AE transducer}

We assume that a transmitter $j$ sends a pulse of amplitude $A_{0}(f)$ to a receiver $i$. In this case, the amplitude $A_{i j}(f)$ recorded by receiver $i$ can be represented by

$$
A_{i j}(f)=S_{i}(f) I_{i}(f) G_{i j}(f) I_{j}(f) S_{j}(f) A_{0}(f),
$$

(Davi et al. 2013; Kwiatek et al. 2014), where $S_{i}$ and $S_{j}$ are coefficients reflecting the coupling condition between the specimen and the transducer (i.e. the transmitter and the receiver). $G_{i j}$ is a Green's function between the transmitter and the receiver. Under the assumption of an isotropic homogeneous medium, this can be represented by $G_{i j} \propto 1 / R_{i j}$, where $R_{i j}$ is the distance between the transmitter and the receiver. $I_{i}$ and $I_{j}$ are the AE transducer responses including directivity. According to Kwiatek et al. (2014), we assume bell-shaped curves

$I_{i} \propto \exp \left(-a \alpha_{i}^{b}\right), \quad I_{j} \propto \exp \left(-a \beta_{j}^{b}\right)$

for $I_{i}$ and $I_{j}$, where $\alpha$ and $\beta$ denote the angles between the ray path of the wave and the transducer (i.e. an incident angle for a receiver and a projection angle for a transmitter). Coefficients $a$ and $b$ determine the shape of the functions. A common function (i.e. the same $a$ and $b$ ) is assumed, regardless of whether the $\mathrm{AE}$ transducer is used as a transmitter or a receiver. Precisely speaking, eqs (1) and (2) are functions of frequency, but we ignore the frequency dependency for simplicity. From eqs (1) and (2), the observed amplitudes $A_{i j}$ recorded by the receiver can be represented by

$A_{i j}=\frac{1}{R_{i j}} \exp \left(-\mathrm{a} \alpha^{b}\right) \exp \left(-\mathrm{a} \beta^{b}\right) S_{i} S_{j} A_{0}$.

To determine $a$ and $b$ for the R15 transducer, we conducted a test using an aluminium block in the shape of a semi-circular column. We attached an R15 transducer to the bottom of the block and measured the amplitudes recorded by the receiver for waves radiated from a transmitter attached to the lateral face of the aluminium block at $0^{\circ}$, 

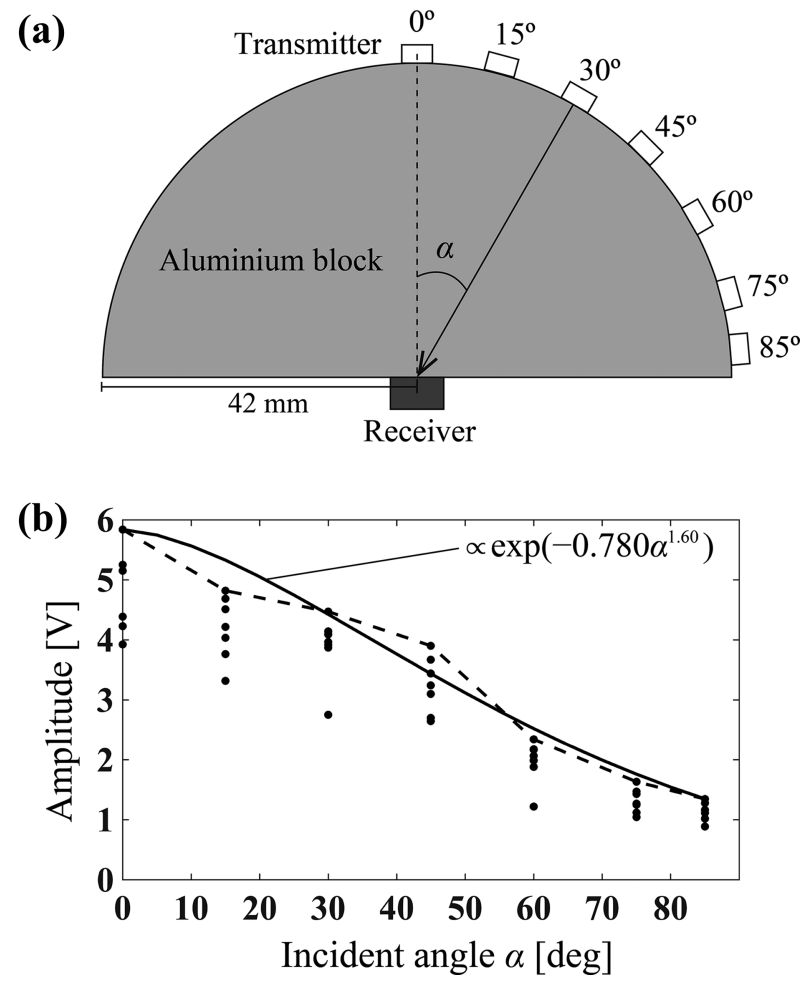

Figure 4. (a) Schematic illustration of the measurement setting for $\mathrm{AE}$ sensor directivity. The receiver is attached to the bottom and transmitters are attached to the lateral face of the block at $0^{\circ}, 15^{\circ}, 30^{\circ}, 45^{\circ}, 60^{\circ}, 75^{\circ}$ and $85^{\circ}$. (b) Measurement results. Dots represent the amplitude of $P$-wave first motions obtained for eight tests at each angle. The dashed line connects the maximum amplitudes at each angle, and the bold line represents the fitting result for the data.

$15^{\circ}, 30^{\circ}, 45^{\circ}, 60^{\circ}, 75^{\circ}$ and $85^{\circ}$, as shown in Fig. 4(a). Because the transmitter must be attached to the curved surface, we used a small transmitter of $4 \mathrm{~mm}$ in diameter (Pico sensor, produced by Physical Acoustics Corp.) to ensure an appropriate coupling condition. The transmitters and the receiver were attached by thermofusible wax, same as the experimental setting of hydraulic fracturing (Section 3). The amplitude recorded by the receiver is affected by the coupling condition of the transmitters and the receiver, and is often reduced by, for example, the thick layer of the wax or the small area of adhesive. Therefore, we conducted the pulse radiation test eight times at each angle, reattaching the transmitter each time. We took the maximum amplitude $A_{k}$ at each incident angle $\alpha_{k}$ to ensure a proper coupling condition, and conducted the subsequent analysis under the assumption that $S_{j}$ remains constant. As for the receiver, we can assume that $S_{i}$ is constant because we did not reattach it during the test.

As shown in Fig. 4(a), the distance between the transmitter and the receiver $R_{i j}$ is a constant and $\beta=0$ for all tests at each angle. Under these conditions, eq. (3) becomes

$A_{k} \propto \exp \left(-a \alpha_{k}^{b}\right)$

We estimated the unknown constants $a$ and $b$ by fitting eq. (4) to the observed $A_{k}$ using the Levenberg-Marquardt method (More 1978). Fig. 4(b) shows the fitting result. The measured amplitudes are explained very well by the obtained curve.
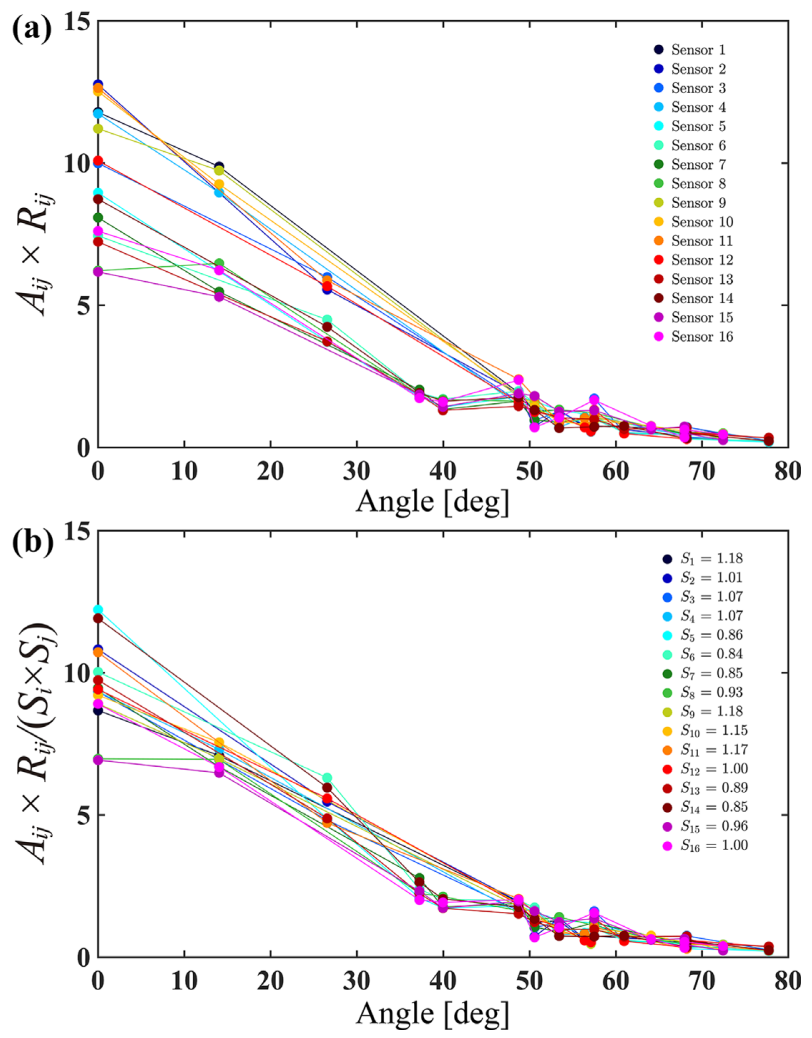

Figure 5. (a) Raw amplitudes recorded at each receiver in the sensor calibration test for KJG07 (Section 4.2). Colours represent the difference at the recording receiver. Records for a common receiver are connected by a single-colour line. The horizontal axis is taken for the average of an incident angle of a receiver and a projection angle of a transmitter. (b) Same plot for the amplitudes, corrected for coupling coefficients.

\subsection{Calibration of coupling conditions}

We estimated the coupling coefficients $S_{i}$ and $S_{j}$ for each fracturing experiment using the following procedure, which is the modified method suggested by Davi et al. (2013) and Kwiatek et al. (2014).

After attaching the AE transducers and applying a $5 \mathrm{MPa}$ load, as shown in Fig. 1, we conducted pulse radiation tests in which test pulses radiated by one of the R15 transducers were recorded by the other AE transducers. $S_{i}$ and $S_{j}$ were estimated from the measured $P$-wave amplitudes. Although $240(15 \times 16)$ waveforms were obtained, we discarded the data in which the transmitter and receiver were attached in the same plane because $\alpha$ and $\beta$ become $90^{\circ}$ and the transducer has little sensitivity. For the remaining 192 amplitude data, $S_{i}$ and $S_{j}$ were estimated using Levenberg-Marquardt inversion on the basis of eq. (3), where the directivity with respect to $\alpha$ and $\beta$ was obtained, as described in the previous section. In the inversion, we constrained the average value of $S_{i}$ across all 16 transducers to be unity. Because hydraulic fracturing was conducted without reattaching the transducers after each test, the obtained relative sensitivities can be used to analyse the AEs during the fracturing experiment.

Fig. 5 represents the amplitudes obtained in this test and the resultant $S_{i}$ and $S_{j}$ for experiment KJG07 (Type V). In Fig. 5(a), which represents the amplitude data before the correction of $S_{i}$ and $S_{j}$, the recorded amplitudes vary at each angle. This scattering is suppressed after the correction, as shown in Fig. 5(b).

In Fig. 5(a), the obtained amplitudes seem to be separated into two groups at lower incident angles. This tendency was recognized only for Type V specimens and reflects the smaller amplitudes of 

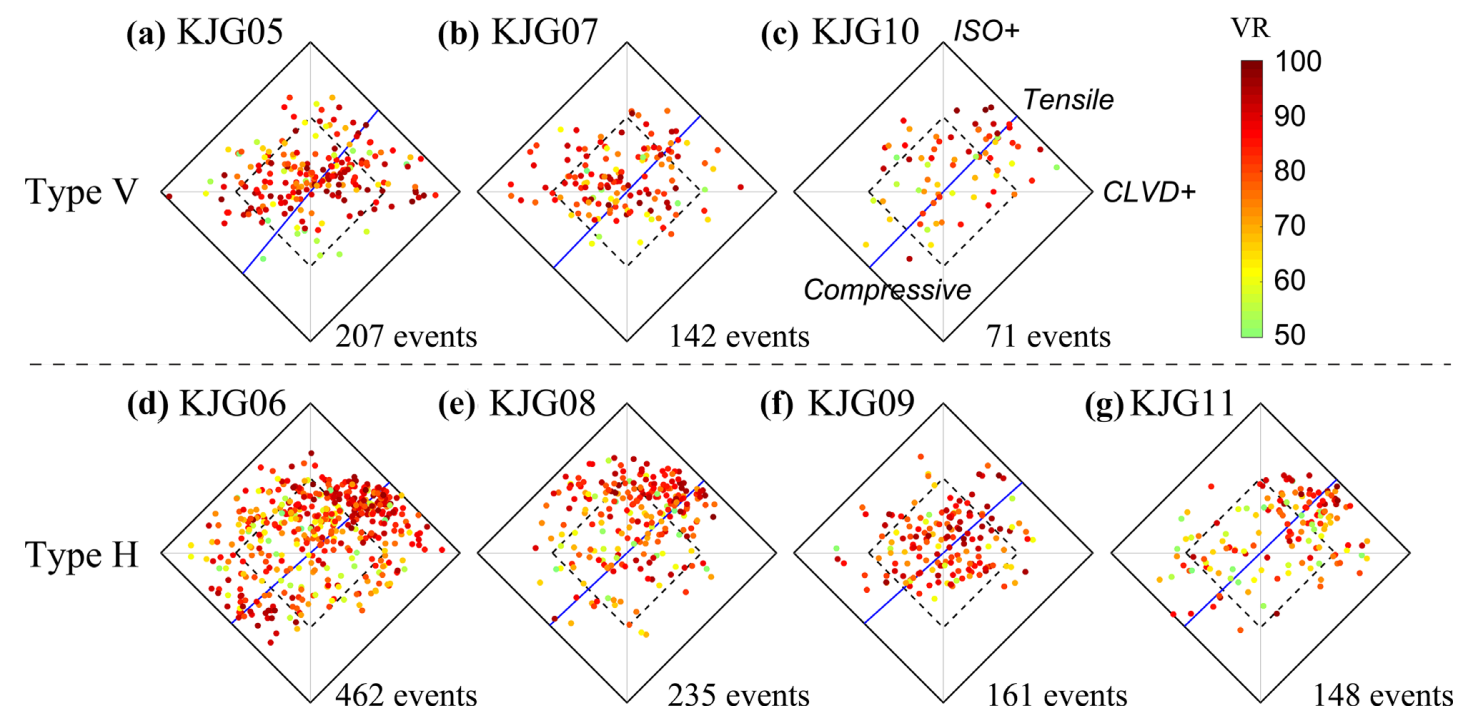

Figure 6. (a-g) Source-type plot for the moment tensors obtained for each experiment. Solutions in the square area surrounded by the dashed lines correspond to events having double couple components of 50 per cent or more (i.e. $|c| \leq 0.5$ ). Colours represent VR of each solution. Ideal shear-tensile events (Vavryčuk 2001, 2011b) correspond to the solutions on the blue line, which are determined by the ratio of $V_{\mathrm{P}}$ to $V_{\mathrm{S}}$ (Vavryčuk 2015). Results for (a) KJG05 (Type V), (b) KJG07 (Type V), (c) KJG10 (Type V), (d) KJG06 (Type H), (e) KJG08 (Type H), (f) KJG09 (Type H) and (g) KJG11 (Type H).

the data having travel paths along the $y$-axis, possibly indicating the large attenuation of the rift plane. Although such anisotropy of attenuation may increase errors in moment tensor estimation (Stierle et al. 2016), the influence was likely reduced by the correction of coupling conditions, on which the effect of anisotropic attenuation was imposed in this study.

\subsection{Moment tensor inversion}

We estimated full moment tensors (i.e. six independent components were estimated) for the AEs in each experiment using the polarities and amplitudes of the initial $P$-wave motions. The relation between $P$-wave amplitudes and a moment tensor can be written as

$U=G M$,

(e.g. Stein \& Wysession 2003), where $U$ is the $n \times 1$ matrix of $P$-wave amplitudes including polarities, $G$ is the $n \times 6$ matrix containing the $P$-wave amplitudes at each channel due to each moment tensor component (related to spatial derivatives of the point force Green's functions that gives displacement seismogram; e.g. Aki \& Richards 2002), $M$ is the $6 \times 1$ matrix containing six independent components of a moment tensor and $n$ is the number of phases used in the inversion. The moment tensor can be estimated by linear inversion based on eq. (5).

In this analysis, the amplitudes were used after the correction of the transducer directivity and coupling conditions. The polarity response of the transducer was verified through a ball-drop experiment, and the independence of the incident angles was also verified in the test described in Section 4.1. For the obtained solutions, we estimated the variance reduction (VR) as

$\mathrm{VR}=\left(1-\frac{\sum\left|A-A^{\text {th }}\right|^{2}}{\sum|A|^{2}}\right) \times 100(\%)$,

where $A$ is the amplitude of the $P$ wave after the correction of coupling and incident angle effects and $A^{\text {th }}$ is the theoretical amplitude. The following analysis considers solutions for which VR $\geq 50$ per cent.
In the above procedure, moment tensors can be estimated by linear inversion when $P$-wave polarities were obtained at all channels. However, the non-linear equation for absolute amplitudes is necessary when the polarities in some channels could not be read due to, for example, low signal-to-noise ratios. To avoid the nonlinear inversion, we inversely estimated multiple solutions by applying both plus and minus polarities to the observed absolute amplitudes at such channels (i.e. the inversion is conducted $2^{\mathrm{m}}$ times when the polarities could not be read at $m$ channels) and adopted the solution with the minimum amplitude residuals. For the channels of $P$-wave polarities unavailable, we used absolute amplitudes in a window of $5 \mu$ s in length from the $P$-wave onsets.

Fig. 6 shows source-type plots of the obtained moment tensor solutions for experiments KJG05-11. The events whose $P$-wave polarities could be read at six or more channels were analysed. Although various source-type plots have been suggested in previous studies, we adopted the 'simplified decomposition method' (Vavryčuk 2015) in accordance with the recommendation of Aso et al. (2016), who compared 13 different source-type plots. In these plots, solutions near the origin correspond to shear events that have large double couple (DC) components, and the DC percentage declines with distance from the origin. Shear-tensile events (Vavryčuk 2001, 2011b), in which both tensile and shear displacement occur on a single fault plane, correspond to solutions along a line determined by the ratio of $V_{\mathrm{P}}$ to $V_{\mathrm{S}}$ (Vavryčuk 2015). The line estimated from the average $V_{\mathrm{P}}$ and $V_{\mathrm{S}}$ among the $x y z$ directions is shown in blue in Fig. 6. On this blue line, the part in the first quadrant corresponds to tensile events (crack opening), whereas the part in the third quadrant corresponds to compressive events (crack closure).

Although shear, tensile and compressive AE events occurred in both Type $\mathrm{V}$ and Type $\mathrm{H}$ samples (see Fig. 6), the shear-type was dominant for Type V (KJG05, 07, 10; Figs 6a-c), whereas the tensile-type was dominant for Type H (KJG06, 08, 11; Figs 6d, e, g). Many compressive events were also observed for Type $\mathrm{H}$, especially for KJG06. An exceptional case is KJG09 (Type H; Fig. 6f), where the fluid pressure parameters discussed in Naoi et al. (2018) display similar characteristics to those in Type V. We discuss the results for KJG09 in Section 5. 
(a) $\mathrm{KJG06}$

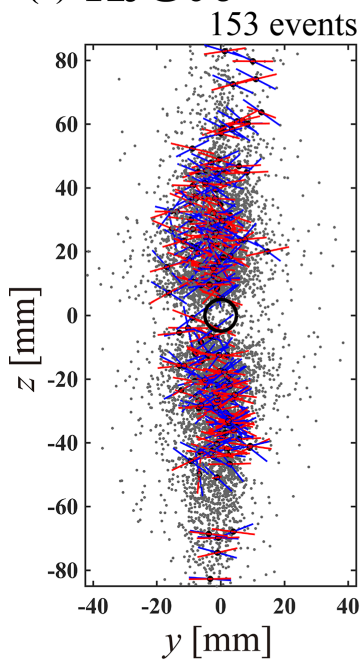

(e)

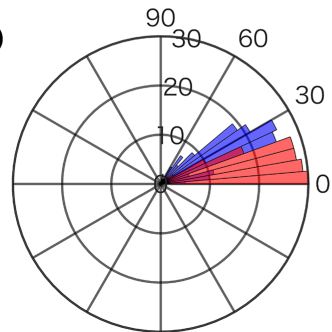

(b) KJG08

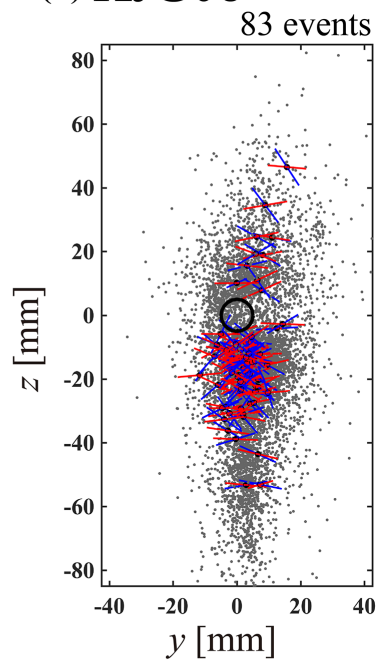

(f)

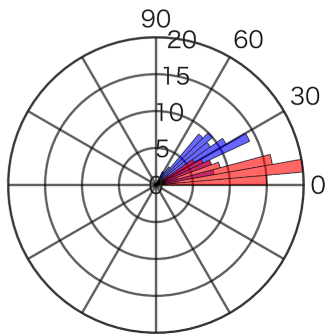

(c) KJG09

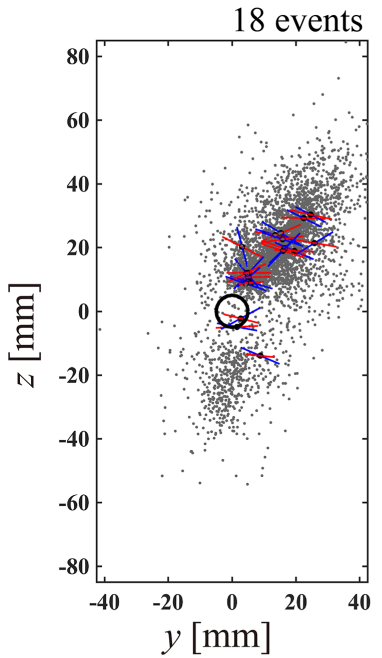

(g)

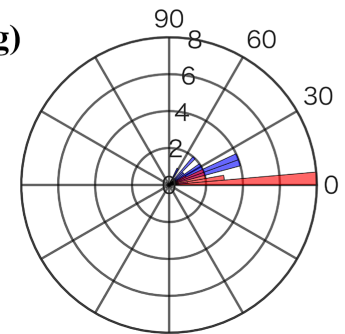

(d) KJG11

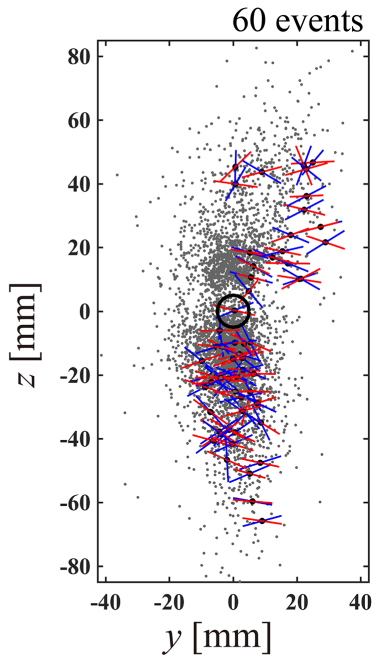

(h)

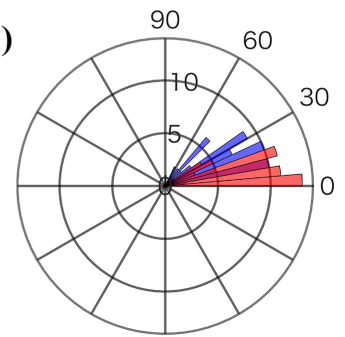

Figure 7. (a-d) $y-z$ projections of the fault normal direction $\boldsymbol{n}$ and dislocation direction $\boldsymbol{v}$ for experiments of Type H specimens. $\boldsymbol{n}$ and $\boldsymbol{v}$ are plotted for the moment tensors that have $c>0.5$ and are situated in the first quadrant of the source-type plot (i.e. tensile-dominant events). The number of such events is shown above each panel. For each pair of $\boldsymbol{n}$ and $\boldsymbol{v}$, the vector with a higher dip angle is shown by blue and another by red (red bins overlay blue ones). Hypocentres relocated by the double difference method, including events whose moment tensor was not determined, are overlaid (grey dots). Results for (a) KJG06, (b) KJG08, (c) KJG09 and (d) KJG11. (e-h) Polar coordinate histogram of dip angles of $\boldsymbol{n}$ and $\boldsymbol{v}$. Results for (e) KJG06, (f) KJG08, (g) KJG09 and (h) KJG11.

\subsection{Crack orientations and instability coefficients}

For the obtained moment tensors, we evaluated the crack orientations under the assumption of the shear-tensile model. For this purpose, we chose solutions using the consistency coefficient (Vavryčuk 2011b)

$c=\operatorname{sign}\left(\frac{\mathrm{ISO}}{\mathrm{CLVD}}\right)\left|1-\frac{\mathrm{DC}}{100}\right|$,

where ISO, CLVD and DC are the percentages of each component after the decomposition. $c$ takes a value between -1 and 1 , and a moment tensor solution is consistent with the shear-tensile model if $c \geq 0$ (Vavryčuk 2011b). $c$ approaches 0 when the shear component becomes large, and approaches 1 when the tensile or compressive component becomes large. In the following analysis, we consider events in which $c \geq 0$.

For the selected events, we calculated the fault normal direction $n$ as

$\boldsymbol{n}=\sqrt{\frac{M_{1}-M_{2}}{M_{1}-M_{3}}} \boldsymbol{e}_{1}+\sqrt{\frac{M_{3}-M_{2}}{M_{3}-M_{1}}} \boldsymbol{e}_{3}$,

and the dislocation direction $\boldsymbol{v}$ as

$\boldsymbol{v}=\sqrt{\frac{M_{1}-M_{2}}{M_{1}-M_{3}}} \boldsymbol{e}_{1}-\sqrt{\frac{M_{3}-M_{2}}{M_{3}-M_{1}}} \boldsymbol{e}_{3}$,

according to Vavryčuk (2011b), where $M_{1}, M_{2}$ and $M_{3}$ are the maximum, intermediate and minimum eigenvalues of a moment tensor, respectively. Eigenvectors $\boldsymbol{e}_{1}$ and $\boldsymbol{e}_{3}$ correspond to $M_{1}$ and
$M_{3}$, respectively. $\boldsymbol{n}$ and $\boldsymbol{v}$ are complementary solutions, such as the nodal planes of a DC component, and cannot be determined uniquely (i.e. two candidates of a source model exist for a pair of $\boldsymbol{n}$ and $\boldsymbol{v})$.

Figs 7(a)-(d) represent the $y-z$ projections of the $\boldsymbol{n}$ and $\boldsymbol{v}$ directions of tensile-dominant events, which are defined as solutions exhibiting $c>0.5$ and situated within the first quadrant of the source-type plot. Polar coordinate histograms of the dip angles of the events are shown in Figs 7(e)-(h). The same diagrams for the compressive events ( $c>0.5$ and situated in the third quadrant) are shown in Fig. 8. For such solutions having large values of $c$, the uncertainty of complementary planes is not problematic because $\boldsymbol{n}$ and $\boldsymbol{v}$ have a similar direction. Most instances of $\boldsymbol{n}$ and $\boldsymbol{v}$ for the tensile and compressive events are subparallel to the horizontal $(x-y)$ plane. This indicates that most of the tensile/compressive events had a fracture plane along the loading axis (z-axis). The tensile events in this orientation are consistent with the conventional theory of hydraulic fracturing, and the compressive events likely correspond to the closure of the opened cracks.

Fig. 9 also represents the same diagram for shear-dominant events (i.e. $0 \leq c \leq 0.5$ ). Although the uncertainty of dip angles owing to the complementary planes becomes larger for the solutions having small values of $c$, the tendency of higher dip angles can be recognized for those events.

For the estimated crack orientations, we calculated fault instability coefficient I (Vavryčuk et al. 2013; Vavryčuk 2014) as

$I=\frac{\tau-\mu\left(\sigma-\sigma_{1}\right)}{\tau_{c}-\mu\left(\sigma_{c}-\sigma_{1}\right)}$, 
(a) $\mathrm{KJG} 06$

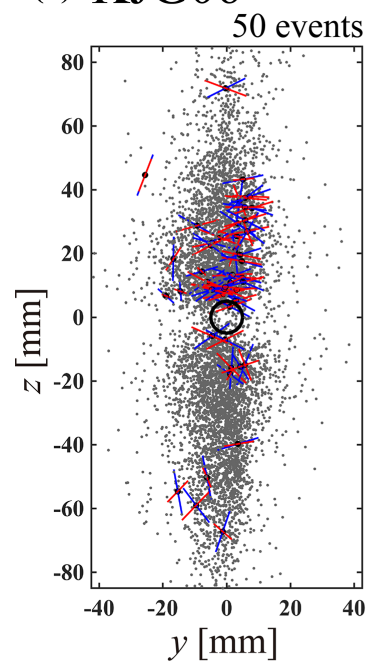

(e)

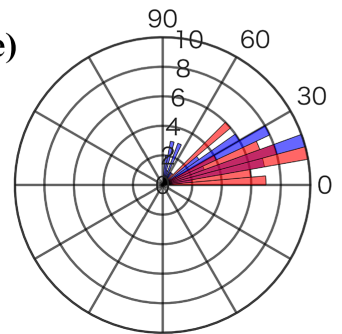

(b) $\mathrm{KJG} 08$

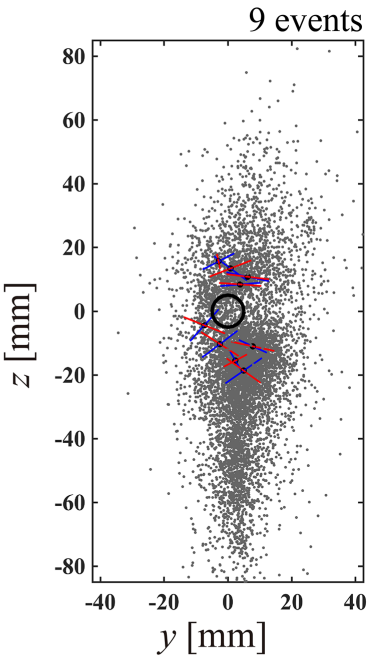

(f)

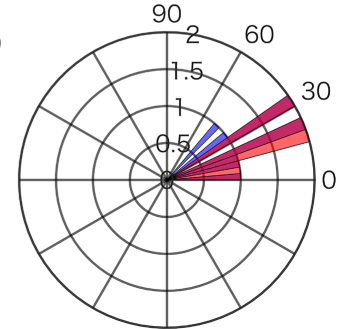

(c) KJG09

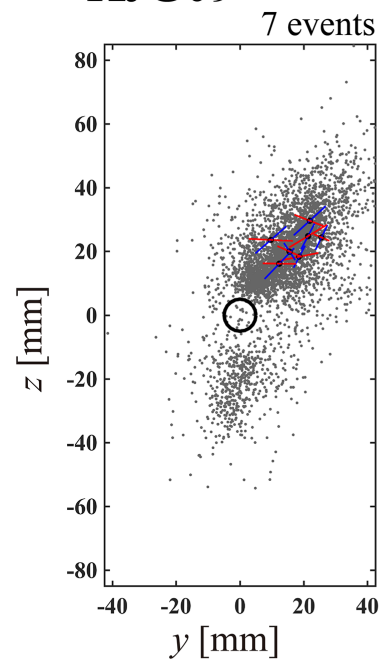

(g)

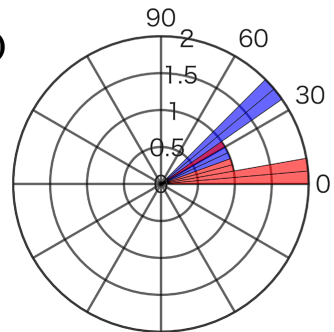

(d) KJG11
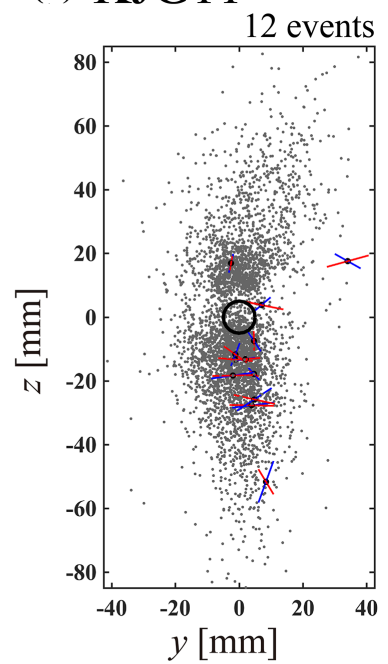

(h)

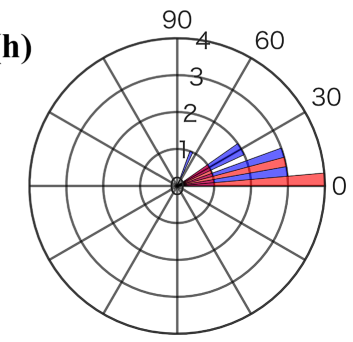

Figure 8. The same diagram as Fig. 7 for the moment tensors that have $c>0.5$ and are situated in the third quadrant of the source-type plot (i.e. compressiondominant events).

\section{(a) $\mathrm{KJG} 06$}

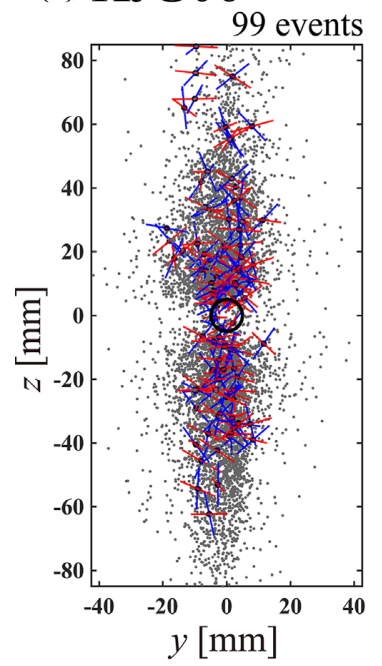

(e)

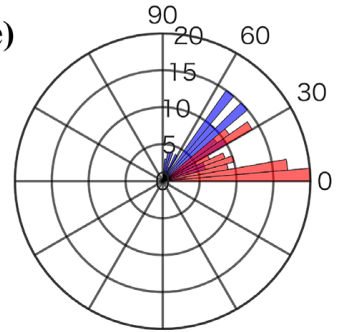

(b) $\mathrm{KJG08}$

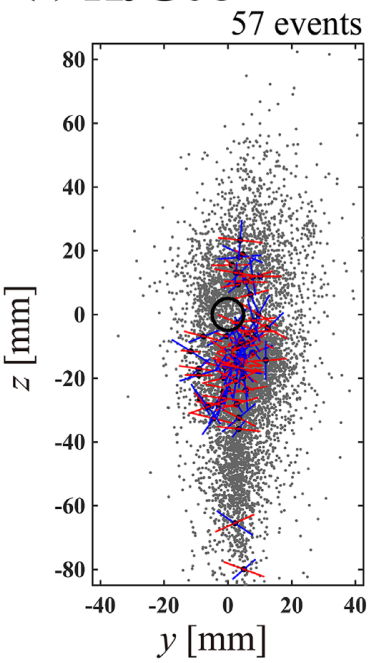

(f)

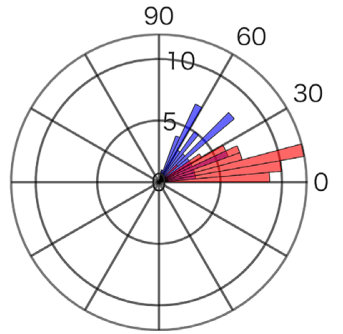

(c) KJG09

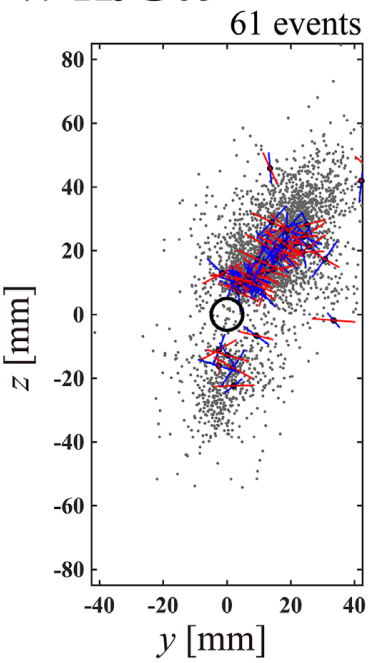

(g)

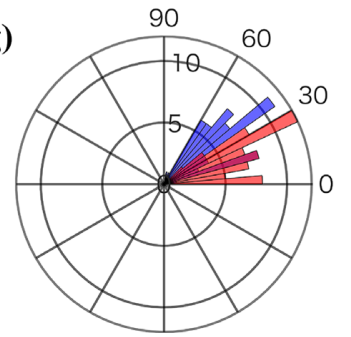

(d) KJG11

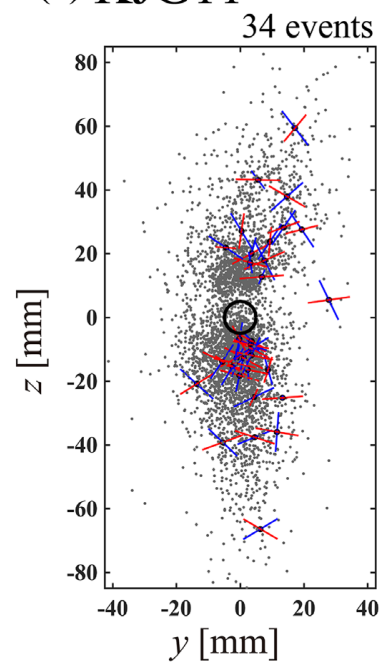

(h)

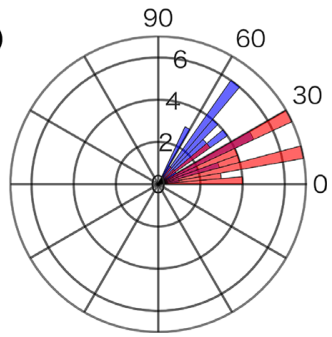

Figure 9. The same diagram as Figs 7 and 8 for the moment tensors that have $0 \leq c \leq 0.5$ (i.e. shear-dominant events). 

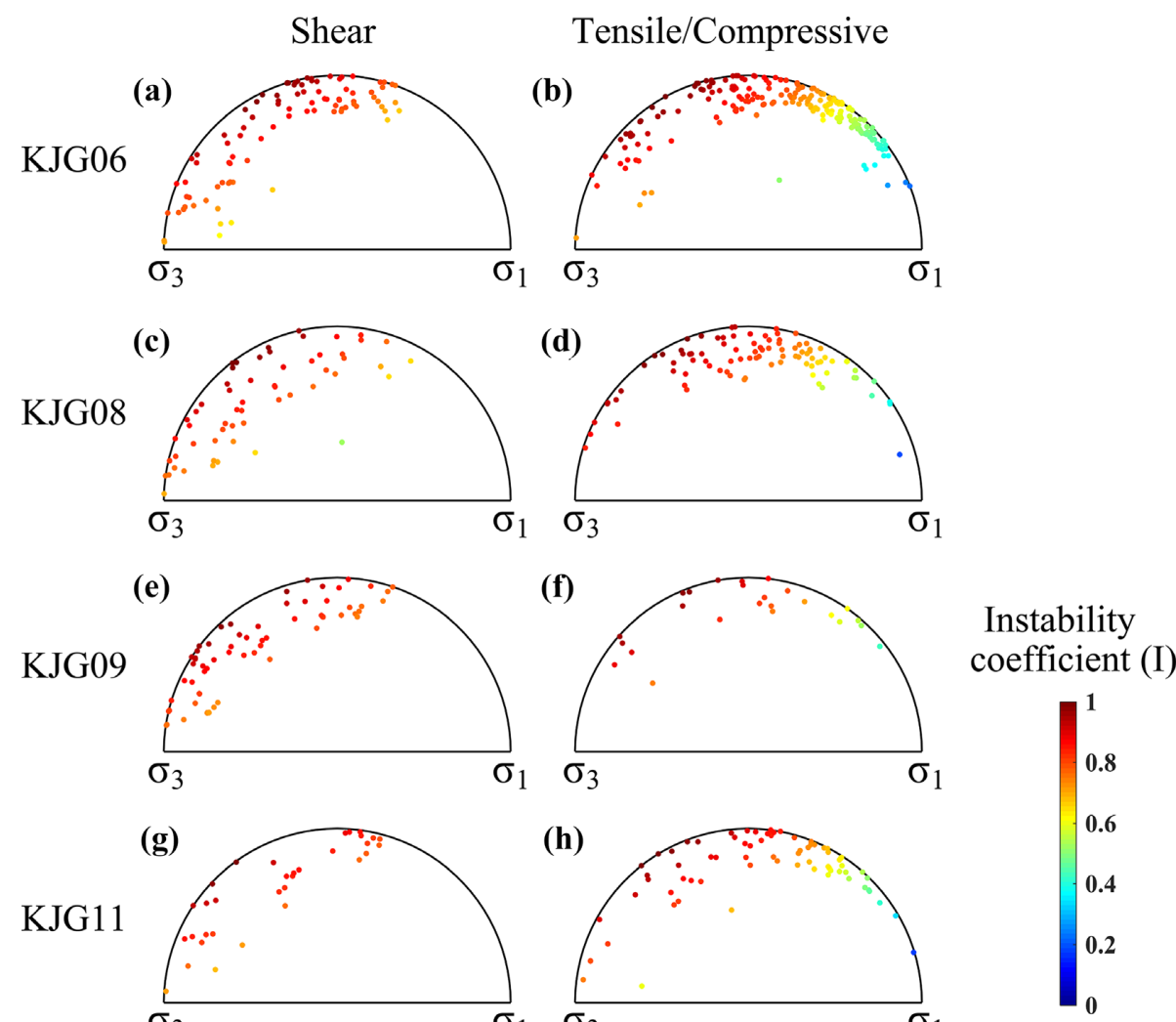

Figure 10. Fault instability coefficient on normalized Mohr's circles. Results for (a) and (b) KJG06, (c) and (d) KJG08, (e) and (f) KJG09, and (g) and (h) KJG11. (a), (c), (e) and (g) represent the results for shear-dominant events with $0 \leq c \leq 0.5$, and (b), (d), (f) and (h) for tensile/compression-dominant events with $c>0.5$.

where $\tau$ and $\sigma$ are shear and effective normal tractions for a crack, $\mu$ is the friction coefficient and $\sigma_{1}$ is the maximum principal stress. $\tau_{c}$ and $\sigma_{c}$ are the shear and effective normal tractions for the "principal fault' (Vavryčuk 2011a), which is the optimally oriented fault in the present stress field. The coefficient varies from 0 for the most stable faults to 1 for the principal fault.

To calculate the instability coefficient, we estimated a stress field around the circular hole under the assumption of the plane strain condition (e.g. Jaeger et al. 2007) where a $5 \mathrm{MPa}$ loading is applied in the $z$-direction. The Poisson ratio, which is necessary to estimate $\sigma_{x x}$, was estimated from the ratio of $V_{\mathrm{P}}$ to $V_{\mathrm{S}}$, and a $\mu$ of 0.6 was assumed. We calculated $I$ for both complementary planes and adopted the one having a larger value of $I$ according to Vavryčuk (2014) and Martínez-Garzón et al. (2016).

Fig. 10 shows the obtained instability coefficient $I$ for Type $\mathrm{H}$ specimens. The normalized Mohr diagram was used to show the shear and normal tractions for each plane according to Vavryčuk (2014). Obviously, shear-dominant events occurred on a plane having larger $I$ than the tensile/compressive-dominant events under the assumed stress fields. For shear-dominant events, a similar range of $I$ was also obtained for Type $\mathrm{V}$ experiments.

\section{DISCUSSION}

As shown in Section 4.3, shear events were dominant in the Type $\mathrm{V}$ experiments and tensile events were dominant for Type H. As described in Section 1, the rift plane orientation of granite can be regarded as the orientation of most of the microcracks. Hence, this dependency indicates that the dominant orientation of pre-existing cracks around a wellbore strongly affects the dominant focal mechanisms induced by hydraulic fracturing.

In this study, the macroscopic direction of an induced fracture and the orientation of the rift plane were orthogonalized for Type $\mathrm{H}$ specimens. In this situation, it is difficult for any interaction between the induced fracture and pre-existing cracks to occur, resulting in the dominance of tensile events along the loading axis, which can be considered a direct result of hydraulic fracturing. This is partly because a propagating crack tends to cross a pre-existing crack when they intersect at a high angle (Blanton 1982; Warpinsky \& Teufel 1987; Potluri et al. 2005), and partly because the vertical loading in this study facilitates the closure of the majority of pre-existing cracks along horizontal $(x-y)$ planes.

We also find many compressive events for Type H. Compressive events were often reported in field observations for hydraulic fracturing/stimulation (Baig \& Urbancic 2010; Martínez-Garzón et al. 2017) and laboratory experiments (Charalampidou et al. 2015). They were explained by, for example, crack compaction by shearing, or the closure of pores/cracks by the decrease of pore pressure due to fluid outflow. As for this case, we consider that the compressive events correspond to the closure of cracks that were opened by tensile events partly because the estimated orientations of the cracks were similar to those of tensile events and partly because they were rare for Type $\mathrm{V}$ samples in which the smaller number of tensile events occurred.

As for Type V samples, the rift plane is parallel to the fracture propagation direction expected from the stress around the wellbore. Although tensile AEs using pre-existing cracks along the rift plane 
are possibly dominant, shear events dominated in the experimental results. One possible explanation for this feature is the influence of aseismic penetration of the fracturing fluid. That is, the pre-existing cracks parallel to the $z$-axis are likely to have been easily opened by the stress around the wellbore, allowing the fracturing fluid to penetrate into the cracks and diffuse sufficiently without causing a sufficient increase in pressure to generate AE events. Indeed, Naoi et al. (2018) observed aseismic regions where fluid penetrated without AEs in their experiments.

Shear events induced during the experiments can be interpreted as the result of the reduction of friction due to an increase in fluid pressure on pre-existing cracks (Pearson 1981; Warpinski et al. 1998; Shapiro et al. 2006) or the results of stress perturbation of hydraulically induced fractures. They are likely easily induced on a plane whose orientation is close to the principal fault (i.e. planes with high instability coefficients). The promotion of shear events on optimally oriented planes was reported in field observations and in situ experiments related to fluid stimulation (Martínez-Garzón et al. 2016; Kwiatek et al. 2018). In this case, the estimated orientation for shear-dominant events, which occurred for both Type H and Type V, showed large instability coefficients. Although such cracks showing large $I$ are likely to have existed before the experiments, they were possibly induced during the experiments by the interaction among cracks along the $\sigma_{1}$ direction, as suggested by previous studies (Hill 1977; Reches \& Lockner 1994).

In experiment KJG09, except for the Type H specimens (Section 4.3), shear events were dominant. In the other Type H specimens, the AEs and the fluid penetration regions extended almost vertically on the macroscopic scale, but that of KJG09 was inclined by $\sim 38^{\circ}$ (see the AE distribution in Figs 7c, 8c, 9c). This may have caused the low number of tensile events. That is, the main and surrounding subsidiary fractures inclined from the maximum compression $\left(\sigma_{1}\right)$ direction cause significant shear stress on the fracture planes, making it difficult to achieve pure tensile opening without shear slip.

\section{CONCLUSIONS}

In this study, we conducted hydraulic fracturing experiments under uniaxial loading conditions in granite blocks where the rift plane is orthogonal (Type H) or parallel (Type V) to the fracture direction that is theoretically expected from the stress field around the wellbore. We monitored the AEs induced by the fracturing and estimated their moment tensors by carefully correcting for the sensor characteristics. For Type H, tensile events along the loading axis were dominant, consistent with the conventional theory of hydraulic fracturing, whereas shear events were dominant for Type V. This difference implies that the dominant focal mechanisms of seismic events induced by hydraulic fracturing are significantly affected by the relation between the directions of both the fracture propagation and pre-existing weak planes around the wellbore.

\section{ACKNOWLEDGEMENTS}

This work was supported financially by Japan Oil, Gas and Metals National Corporation (JOGMEC) and JSPS KAKENHI (Grant number 16H04614).

\section{REFERENCES}

Aki, K. \& Richards, P.G., 2002. Quantitative Seismology, 2nd edn, University Science Books.
Allen, R.V., 1978. Automatic earthquake recognition and timing from single traces, Bull. seism. Soc. Am., 68, 1521-1532.

Aso, N., Ohta, K. \& Ide, S., 2016. Mathematical review on source-type diagrams, Earth Planets Space, 68, doi:10.1186/s40623-016-0421-5.

Baig, A. \& Urbancic, T., 2010. Microseismic moment tensors: a path to understanding frac growth, Leading Edge, 29, 320-324.

Blanton, T.L., 1982. An experimental study of interaction between hydraulically induced and pre-existing fractures, in SPE Unconventional Gas Recovery Symposium, Pittsburgh, PA.

Charalampidou, E., Stanchits, S., Kwiatek, G. \& Dresen, G., 2015. Brittle failure and fracture reactivation in sandstone by fluid injection, Eur. $J$. Environ. Civ. Eng., 19, 564-579.

Davi, R., Vavryčuk, V., Charalampidou, E. \& Kwiatek, G., 2013. Network sensor calibration for retrieving accurate moment tensors of acoustic emissions, Int. J. Rock Mech. Min. Sci., 62, 59-67.

Fischer, T. \& Guest, A., 2011. Shear and tensile earthquakes caused by fluid injection, Geophys. Res. Lett., 38, doi:10.1029/2010GL045447.

Hill, D.P., 1977. A model for earthquake swarms, J. geophys. Res., 82, $1347-1352$

Horálek, J., Jechumtálová, Z., Dorbath, L. \& Šilený, J., 2010. Source mechanisms of micro-earthquakes induced in a fluid injection experiment at the HDR site Soultz-sous-Forêts (Alsace) in 2003 and their temporal and spatial variations, Geophys. J. Int., 181, 1547-1565.

Hubbert, M.K. \& Wills, D.G., 1957. Mechanics of hydraulic fracturing, Trans. Soc. Pet. Eng. AIME, 210, 153-168.

Ishida, T., Chen, Q., Mizuta, Y. \& Roegiers, J.C., 2004. Influence of fluid viscosity on the hydraulic fracturing mechanism, J. Energy Resour. Technol., 126, 190-200.

Jaeger, J.C., Cook, N.G.W. \& Zimmerman, R.W., 2007. Fundamentals of Rock Mechanics, 4th edn, Blackwell.

Julian, B.R., Foulger, G.R., Monastero, F.C. \& Bjornstad, S., 2010. Imaging hydraulic fractures in a geothermal reservoir, Geophys. Res. Lett., 37, doi:10.1029/2009GL040933.

Kwiatek, G., Charalampidou, E., Dresen, E. \& Stanchits, S., 2014. An improved method for seismic moment tensor inversion of acoustic emissions through assessment of sensor coupling and sensitivity to incidence angle, Int. J. Rock Mech. Min. Sci., 65, 153-161.

Kwiatek, G., Martínez-Garzón, P., Plenkers, K., Leonhardt, M., Zang, A., von Specht, S., Dresen, G. \& Bohnhoff, M., 2018. Insights into complex subdecimeter fracturing processes occurring during a water injection experiment at depth in Äspö Hard Rock Laboratory, Sweden, J. geophys. Res., 123, 6616-6635.

Martínez-Garzón, P., Kwiatek, G., Bohnhoff, M. \& Dresen, G., 2016. Impact of fluid injection on fracture reactivation at The Geysers geothermal field, J. geophys. Res., 121, 7432-7449.

Martínez-Garzón, P., Kwiatek, G., Bohnhoff, M. \& Dresen, G., 2017. Volumetric components in the earthquake source related to fluid injection and stress state, Geophys. Res. Lett., 44, 800-809.

Maxwell, S.C. \& Cipolla, C., 2011. What does microseismicity tell us about hydraulic fracturing?, in SPE Annual Technical Conference and Exhibition, Denver, CO.

More, J.J., 1978. The Levenberg-Marquardt Algorithm: implementation and theory, Numer. Anal., 630, 105-116.

Naoi, M. et al., 2018. Monitoring hydraulically-induced fractures in the laboratory using acoustic emissions and the fluorescent method, Int. J. Rock Mech. Min. Sci., 104, 53-63.

Nolen-Hoeksema, R.C. \& Ruff, J.R., 2001. Moment tensor inversion of microseisms from the B-sand propped hydrofracture, M-site, Colorado, Tectonophysics, 336, 163-181.

Pearson, C., 1981. The relationship between microseismicity and high pore pressures during hydraulic stimulation experiments in low permeability granitic rocks, J. geophys. Res., 86, 7855-7864.

Potluri, N.K., Zhu, D. \& Hill, A.D., 2005. The effect of natural fractures on hydraulic fracture propagation, in SPE European Formation Damage Conference, Sheveningen, The Netherlands.

Reches, Z. \& Lockner, D.A., 1994. Nucleation and growth of faults in brittle rocks, J. geophys. Res., 99, 159-173. 
Rothman, R.L., Greenfield, R.J. \& Hardy, H.R., 1974. Errors in hypocenter location due to velocity anisotropy, Bull. seism. Soc. Am., 64, 1993-1996.

Sano, O., Kudo, Y. \& Mizuta, Y., 1992. Experimental determination of elastic constants of Oshima granite, Barre granite, and Chelmsford granite, $J$. geophys. Res., 97, 3367-3379.

Shapiro, S.A., Dinske, C. \& Rothert, E., 2006. Hydraulic-fracturing controlled dynamics of microseismic clouds, Geophys. Res. Lett., 33, doi:10.1029/2006GL026365.

Shimizu, H., Murata, S. \& Ishida, T., 2011. The distinct element analysis for hydraulic fracturing in hard rock considering fluid viscosity and particle size distribution, Int. J. Rock Mech. Min. Sci., 48, 712-727.

Šilený, J., Hill, D.P., Eisner, L. \& Cornet, F.H., 2009. Non-double-couple mechanisms of micro earthquakes induced by hydraulic fracturing, $J$. geophys. Res., 114, doi:10.1029/2008JB005987.

Šilený, J., Jechumtálová, Z. \& Dorbath, C., 2014. Small scale earthquake mechanisms induced by fluid injection at the enhanced geothermal system reservoir Soultz (Alsace) in 2003 using alternative source models, Pure appl. Geophys., 171, 2783-2804.

Staněk, F. \& Eisner, L., 2017. Seismicity induced by hydraulic fracturing in shales: a bedding plane slip model, J. geophys. Res., 122, 7912-7926.

Stein, S. \& Wysession, M., 2003. An Introduction to Seismology, Earthquakes, and Earth Structure, Blackwell Publishing.

Stierle, E., Vavryčuk, V., Kwiatek, G., Charalampidou, E. \& Bohnhoff, M., 2016. Seismic moment tensors of acoustic emissions recorded during laboratory rock deformation experiments: sensitivity to attenuation and anisotropy, Geophys. J. Int., 205, 38-50.

Takanami, T. \& Kitagawa, G., 1988. A new efficient procedure for the estimation of onset times of seismic waves, J. Phys. Earth., 36, 267-290.
Vavryčuk, V., 2001. Inversion for parameters of tensile earthquakes, J. geophys. Res., 106, 16339-16355.

Vavryčuk, V., 2002. Non-double-couple earthquakes of 1997 January in West Bohemia, Czech Republic: evidence of tensile faulting, Geophys. J. Int., 149, 364-373.

Vavryčuk, V., 2011a. Principal earthquakes: theory and observations for the 2008 West Bohemia swarm, Earth planet. Sci. Lett., 305, 290-296.

Vavryčuk, V., 2011b. Tensile earthquakes: theory, modeling and inversion, J. geophys. Res., 116, doi:10.1029/2011JB008770.

Vavryčuk, V., Bouchaala, F. \& Fischer, T., 2013. High-resolution fault image from accurate locations and focal mechanisms of the 2008 swarm earthquakes in West Bohemia, Czech Republic, Tectonophysics, 590, 189-195.

Vavryčuk, V., 2014. Iterative joint inversion for stress and fault orientations from focal mechanisms, Geophys. J. Int., 199, 69-77.

Vavryčuk, V., 2015. Moment tensor decomposition revisited, J. Seismol., 19, 231-252.

Waldhauser, F. \& Ellsworth, L.W., 2000. A double difference earthquake location algorithm: method and application to the Northern Hayward fault, California, Bull. seism. Soc. Am., 90, 1353-1368.

Warpinsky, N.R. \& Teufel, L.W., 1987. Influence of geologic discontinuities on hydraulic fracture propagation, J. Pet. Technol., 39, doi:10.2118/13224PA.

Warpinski, N.R., Branagan, P.T., Wolhart, S.L. \& Uhl, J.E., 1998. Mapping hydraulic fracture growth and geometry using microseismic events detected by a wireline retrievable accelerometer array, in SPE Gas Technology Symposium, Calgary, AB.

Yew, C.H. \& Weng, X., 2014. Mechanics of Hydraulic Fracturing, Gulf Professional Publishing. 